\title{
Modeling of Charge Transport in Ion Bipolar Junction Transistors
}

\author{
Anton Volkov, Klas Tybrandt, Magnus Berggren and Igor Zozoulenko
}

\section{Linköping University Post Print}

\section{Tweet}

N.B.: When citing this work, cite the original article.

Original Publication:

Anton Volkov, Klas Tybrandt, Magnus Berggren and Igor Zozoulenko, Modeling of Charge Transport in Ion Bipolar Junction Transistors, 2014, Langmuir, (30), 23, 6999-7005.

http://dx.doi.org/10.1021/la404296g

Copyright: American Chemical Society

http://pubs.acs.org/

Postprint available at: Linköping University Electronic Press

http://urn.kb.se/resolve?urn=urn:nbn:se:liu:diva-109131 


\title{
Modeling of Charge Transport in Ion Bipolar Junction Transistors
}

\author{
Anton V. Volkov, ${ }^{*}$ Klas Tybrandt, ${ }^{*}$ Magnus Berggren, ${ }^{*}$ and Igor V. Zozoulenko* \\ Laboratory of Organic Electronics, ITN, Linköping University, 60174 Norrköping, Sweden \\ E-mail: anton.volkov@liu.se; klas.tybrandt@liu.se; magnus.berggren@liu.se; \\ igor.zozoulenko@liu.se
}

\begin{abstract}
Spatiotemporal control of the complex chemical microenvironment is of great importance to many fields within life science. One way to facilitate such control is to construct delivery circuits, comprising arrays of dispensing outlets, for ions and charged biomolecules based on ionic transistors. This allows for addressability of ionic signals which opens up for spatiotemporally controlled delivery in a highly complex manner. One class of ionic transistors, the ion bipolar junction transistors (IBJTs), is especially attractive for these applications since these transistors are functional at physiological conditions and have been employed to modulate the delivery of neurotransmitter to regulate signalling in neuronal cells. Further, the first integrated complementary ionic circuits were recently developed based on these ionic transistors. However, detailed understanding of the device physics of these transistors is still lacking and hampers further development of components and circuits. Here we report on the modeling of IBJTs using the Poisson's-Nernst-Planck equations and the finite element method. A two-dimensional model of the device is employed that successfully reproduces the main characteristics of the measurement data. Based on the detailed concentration and potential profiles provided by the model, the different modes of operation of the transistor are analyzed as well as the transitions between the different modes. The model correctly predicts the measured threshold voltage, which is explained in terms of membrane potentials. All in all, the results provide the basis for detailed understanding of IBJT operation. This new knowledge is employed to discuss potential improvements of ion bipolar junction transistors in terms of miniaturization and device parameters.
\end{abstract}

\section{Introduction}

Ionic transistors ${ }^{1-3}$ have received significant attention during recent years, much due to envisioned applications within biotechnology and life science. ${ }^{4}$ In ionic transistors the charge carriers are represented by ions and charged biomolecules, which themselves possess chemical and biological information and can therefore be utilized to regulate and stimulate various systems at high specificity. The most common approach to realize ionic transistors is to control the surface charge in nanocavities, ${ }^{5}$ which has been explored in nanofluidic transistors. ${ }^{1,6}$ However, this approach has severe limitations with respect to electrolyte concentration as the Debye screening length at $0.1 \mathrm{M}$ is $\sim 1$ $\mathrm{nm}$. This is problematic since many of the targeted applications require physiological conditions. An alternative approach to the field-effect mode of operation in nanofluidics is to utilize ion exchange membranes (IEMs), ${ }^{7}$ which possess ion selectivity within the bulk phase of the material. The selectivity to either cations or anions in these polymer membranes is a consequence of fixed ionic groups anchored to the polymer matrix. The fixed charges are compensated by mobile counterions of the opposite charge and exclude mobile coions of the same charge (Donnan exclusion). ${ }^{8}$ Cation- 
and anion-selective IEMs are the ionic equivalents to $p$-and $n$-doped semiconductors, respectively. ${ }^{9}$ Based on these materials we have recently reported on the development of ion bipolar junction transistors (IBJTs), ${ }^{3,10}$ the ionic equivalents to the conventional electronic bipolar junction transistors. ${ }^{11}$ In similarity to the electronic counterpart, both pnp- and npn-IBJTs can be constructed. IBJTs are attractive since they are functional at physiological conditions, which has been utilized to modulate the transport of neurotransmitter to cultured neuronal cells. ${ }^{3}$ Also, the first integrated ionic logic circuits were recently reported. ${ }^{13}$

This work is focused on the understanding of the ion transport in the $n p n$-IBJT. ${ }^{9}$ A qualitative model of the ion transport in IBJTs has previously been proposed based on the known characteristics of IEMs and bipolar membranes ${ }^{9}$ (a stack of an anion and a cation-selective membrane). The transport theory of these systems is well developed due to their applications in separation and desalination. ${ }^{9,13-15}$ However, transport in membranes typically occurs in one dimension while the transport in IBJTs must be treated as (at least) a two dimensional problem. Therefore the results from membrane science are not applicable to IBJTs in order to get a comprehensive model. The lack of precise understanding of the transport processes in IBJTs hampers further device and circuit development, for which a detailed theoretical understanding is necessary. It is of particular importance to gain knowledge about how to improve critical device parameters like on-off ratio and switching speed. To address these issues, here we develop a numerical model of the ion transport in IBJTs. The model is based on Nernst-Planck and Poisson's equations, which previously have been successfully applied to IEMs and bipolar membranes in one dimension. ${ }^{15}$ The resulting highly nonlinear system of partial differential equations is solved with the finite element method. In this work, only the steady state solutions are regarded and compared with new and previously published measurement data. The parameters used in the model are estimated from material properties and no fitting to device characteristics is performed.

\section{Physical model}

Our model describes the ion bipolar junction transistor which was developed and experimentally studied by Tybrandt et al. ${ }^{10}$ This $n p n$-IBJT comprises a collector (C), an emitter (E), a base (E) and a neutral junction, (Figure 1a). The emitter and collector are represented by anion-selective membrane channels while the base is a cationselective membrane channel. Each channel is connected to a reservoir filled with $\mathrm{NaCl}$ solution. The reservoirs include conducting poly $(3,4-$ ethylenedioxythiophene):poly(styrenesulfonate) ${ }^{17}$ (PEDOT:PSS) polymer electrodes which are used to convert electric signals into ionic ones. The emitter reservoir is grounded, the collector reservoir has potential $V_{E C}$ and the base reservoir has potential $V_{E B} . V_{E C}$ is the driving voltage for anions to be transported from the emitter to the collector, while $V_{E B}$ modulates the ion concentration within the junction and thereby the ionic current through the collector $\left(I_{C}\right)$. Because of the selectivity of the materials, cations are primarily transported in and out of the base while anions go from the emitter to the collector. The operation of a BJT is typically divided into three regimes. First, when both the emitter-base (E-B) and the collector-base (C-B) diodes are in reverse bias the transistor operates in the cutoff mode. Second, if the E-B diode is in forward bias but the $\mathrm{C}$-B diode is in reverse bias the transistor is in active mode. Thirdly, when the base current is elevated so that the charge carrier concentration at the collector is non-zero, the transistor operates in saturation mode.

It is desirable to reduce the dimensionality of the problem from three dimensions (3D) to two dimensions (2D) in order to decrease the required computational resources. This can be achieved by aligning the base in the same direction as the emitter and collector. The spatial distribution of the ions between the base and junction might be slightly altered, however no major effect is expected as the base current generally is small at steady state conditions. With this modified geometry there should be no substantial ionic fluxes or potential gradients in the $y$ direction, thus a 2D model can be employed as a good approximation. To get the same width $w=200 \mu \mathrm{m}$ for all parts of the transistor, the length and width 


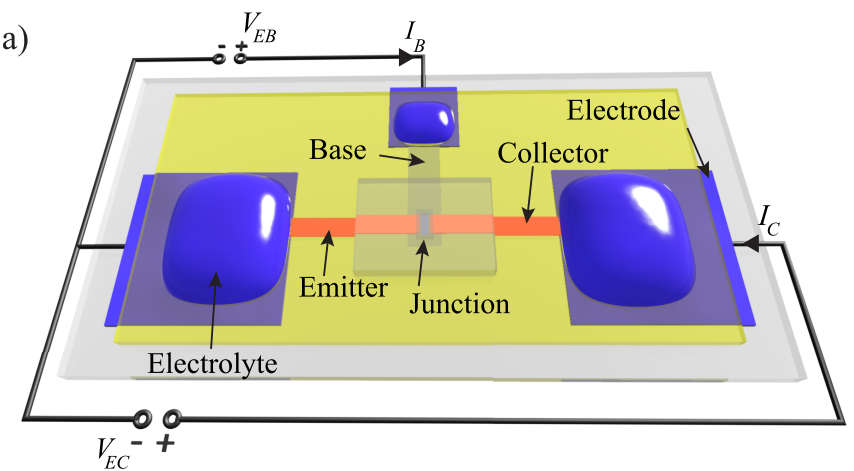

b)

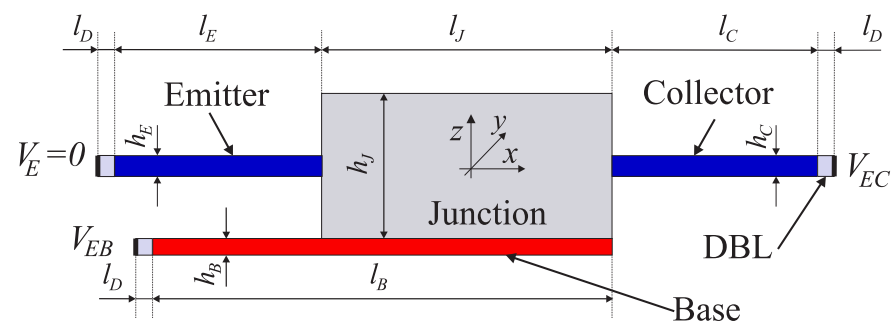

Figure 1: (a) Schematic representation of the IBJT. The emitter, collector and base are connected to the neutral junction. The polymer electrodes covered by electrolytes transfer ions to the terminals of the transistor. (b) The corresponding 2D geometry used in the computer simulation.

of the base is shrunk two times in order to preserve the base resistance. The final 2D geometry is shown in Figure $1 \mathrm{~b}$ with the dimensions given in table Table 1 . The notations $l, h, w$ correspond to the length, height and width whereas indexes $J, B, C, E, D$ correspond to the junction, base, collector, emitter and diffusion boundary layer, respectively.

Table 1: Dimensions used in the computer simulation of the transistor (See Figure 1b)

\begin{tabular}{|l|l|}
\hline & Size $[\mu \mathrm{m}]$ \\
\hline \hline$l_{J}$ & 100 \\
\hline$h_{J}$ & 20 \\
\hline$l_{B}$ & 1350 \\
\hline$h_{B}$ & 0.25 \\
\hline$l_{C}, l_{E}$ & 1450 \\
\hline$h_{C}, h_{E}$ & 0.6 \\
\hline$l_{D}$ & 2 \\
\hline$w$ & 200 \\
\hline
\end{tabular}

The Nernst-Planck equation is employed to model the ion transport in the IBJT

$$
\overrightarrow{j_{i}}=-D_{i}(\mathbf{r})\left[\vec{\nabla} c_{i}+z_{i} f c_{i} \vec{\nabla} V\right]
$$

where $\overrightarrow{j_{i}}$ is the flux of species $i, D(\mathbf{r})$ is the diffusion coefficient, $c_{i}$ is the concentration $\left(c_{+}=c_{N a}\right.$, $\left.c_{-}=c_{C l}\right), z_{i}= \pm 1$ is the valence, $f \equiv F / R T$ ( $F$ is the Faraday constant, $R$ is the molar constant and $T=300 \mathrm{~K}$ is the temperature) and $V$ is the electric potential.

The flux densities are obtained by solving the steady-state equation

$$
\vec{\nabla} \overrightarrow{j_{i}}=0
$$

together with Poisson's equation

$$
-\varepsilon \varepsilon_{0} \triangle V=F\left(c_{+}-c_{-}+z c_{\text {fixed }}(\mathbf{r})\right),
$$

where $c_{\text {fixed }}(\mathbf{r})$ is the concentration of the fixed charges, $z$ is the valence(charge number) of the fixed charges, $\varepsilon_{0}$ is the permittivity of vacuum $\varepsilon$ is the dielectric permittivity of the system with $\varepsilon=40$ everywhere except in the diffusion layers where $\varepsilon=81$.

The reservoirs and electrodes are introduced into the physical model by the concept of the Nernst diffusion layer. According to this concept an unstirred diffusion boundary layer (DBL) is formed at the membrane/solution interface. The solution outside the DBL is assumed to be completely stirred and thus have the concentration of the bulk solution. In the real IBJT the channel interface towards the electrolyte is a large lateral surface which pose no restriction on ion transport over the interface. However, in the model the interface is represented by a small vertical surface instead, which reaches current limiting behavior for even moderate currents. In order to modulate the interfaces of the real IBJT, the width of the DBL has to be small. By setting $l_{D}=2 \mu \mathrm{m}$ both non-limiting behavior and realistic interface concentrations are achieved.

The boundary conditions for eqs 2, 3 are as follows. The concentration for each species is $c_{0}=$ $0.1 \mathrm{M}$ at each border of the diffusion boundary layers. The potential $V_{E B}$ is applied on the left end of the base DBL, $V_{E C}$ is applied on the right end of the collector DBL and $V_{E}=0$ is applied on the left end of the emitter DBL. The potential gradients 
and normal fluxes vanish at all other boundaries

$$
\vec{n} \cdot \vec{\nabla} V=0, \vec{n} \cdot \overrightarrow{j_{i}}=0
$$

The resistance of the channel $\left(R_{S}\right)$ and the diffusion coefficient were obtained from electric measurements on single channels. Unfortunately, the degree of swelling in the devices is unknown and thus the fixed charge density of the materials cannot therefore be exactly estimated. However, by assuming typical fixed charge densities the ion diffusion coefficients can be estimated $\left(z c_{\text {fixed }}=1.2\right.$ $\mathrm{M}$ inside collector and emitter, $z c_{\text {fixed }}=-1.2$ $\mathrm{M}$ inside base, and $c_{\text {fixed }}=0$ within junction and diffusion layers). For a single channel the contribution from counterion migration dominates the transport, thus the diffusion contribution in Nernst-Planck equation can be neglected. Further, by assuming an ideally selective membrane, i.e. only counterions contribute to the current, the current can be written as

$$
I=D c_{\text {fixed }} \frac{F^{2}}{R T} \frac{V h w}{l} .
$$

Ohm's law $I=V / R_{s}$ and eq 5 leads to

$$
D=\frac{R T l}{F^{2} c_{\text {fixed }} R_{s} h w} .
$$

By using eq 6 and the values for the experimental resistance ${ }^{10}$ the diffusion coefficients for the counterions are obtained. The diffusion coefficients for the coions cannot be calculated separately but can be estimated by assuming that the proportionality between the diffusion coefficients for coions and counterions in the membrane is the same as in water. Further, the diffusion coefficients in the junction are assumed to be half of those in water. Estimated values of the diffusion coefficients are summarized in Table 2.

The simulation was performed by using COMSOL 4.3 software. A system of partial differential equations (PDEs) comprising the Poisson's (3) and steady-state (2) equations were written in the general form. A boundary mesh was used for the electric double layers (EDLs) of the channel interfaces. A mapped mesh was used to resolve the extended space charge regions at the collectorjunction and base-junction interfaces. Outside these regions a free triangular mesh was used. Dif-
Table 2: Diffusion coefficients

\begin{tabular}{|l|l|}
\hline & $\mathrm{D}\left[10^{-10} \mathrm{~m}^{2} / \mathrm{s}\right]$ \\
\hline \hline$N a_{\text {water }}^{+}$ & 13.34 \\
\hline$C l_{\text {water }}^{-}$ & 20.32 \\
\hline$N a_{\text {collector,emitter }}^{+}$ & 1.5 \\
\hline$C l_{\text {collector,emitter }}^{-}$ & 2.25 \\
\hline$N a_{\text {base }}^{+}$ & 13.38 \\
\hline$C l_{\text {base }}^{-}$ & 20.39 \\
\hline$N a_{\text {junction }}^{+}$ & 6.67 \\
\hline$C l_{\text {junction }}^{-}$ & 10.16 \\
\hline
\end{tabular}

ferent sizes of the mesh elements were tested in order to ensure the accuracy of the simulation.

The base and collector currents were obtained by line integration over the left end of the base boundary layer and the right end of the collector boundary layer, respectively,

$$
I=F w \int\left(\overrightarrow{j_{+}}-\overrightarrow{j_{-}}\right) d \mathbf{l} .
$$

\section{Results and discussion}

Transfer curve. One of the most important characteristics of the transistor is the transfer curve, which is obtained by measuring the collector current for different $V_{E B}$. Figure 2 shows simulated curves alongside measured data for $-1<V_{E B}<3$ $\mathrm{V}$ and $V_{E C}=10 \mathrm{~V}$. Two regimes are visible, as $I_{C}$ is low for $V_{E B} \lesssim-0.25 \mathrm{~V}$ and depends linearly on $V_{E B}$ for $V_{E B} \gtrsim 0$. The simulated curves are in good agreement with the measurement data. The measured base current is lower than the calculated one, which probably is due to field-enhanced water splitting at the junction-collector interface, an effect well-known for amine-containing IEMs. ${ }^{18}$ This may also be the cause of the higher leakage current in the measured data for $V_{E B}=-1 \mathrm{~V}$ compared to the simulated data. Since the simulated data does not take water splitting into account, $I_{C}$ for $V_{E B}=-1 \mathrm{~V}$ constitutes a lower limit for the leakage current. The simulated on-off ratio $\left(I_{C}(3) / I_{C}(-1)\right)$ is 24000 compared to 31 for the real device. Thus, by suppressing water splitting 18 the simulation indicates that the on-off ratio can be greatly improved.

When the applied potential $V_{E B} \lesssim-0.25 \mathrm{~V}$ the 


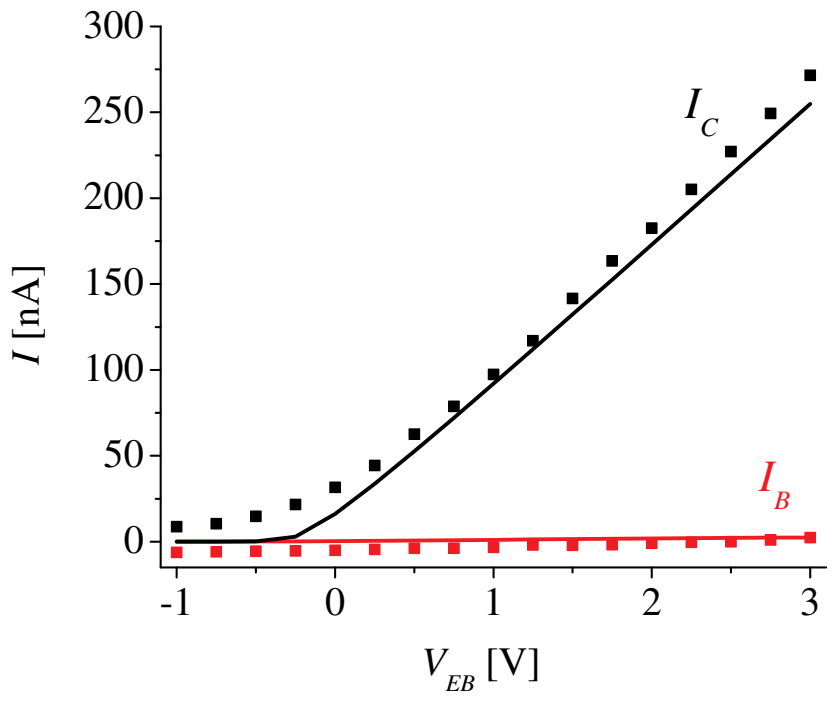

Figure 2: The transfer curves are given for simulated data (line) and experimental data ${ }^{10}$ (square) for $V_{E C}=10 \mathrm{~V}$. $I_{C}$ depends linearly on emitterbase voltage in the active mode while $I_{B}$ remains low.

transistor is in cutoff mode (Figure 3a-d). In this regime there are very few mobile ions within the junction (Figure $3 b$ ) and the whole potential drop occurs at the collector-junction interface (Figure 3c). The low $I_{C}$ in this mode is a consequence of that the potential drop occurs in a region of low conductivity (junction) while the potential is flat in highly conductive regions (emitter and collector). Interestingly, an asymmetric effect can be seen in in the potential distribution in Figure 3d. Even though the junction is essentially non-conducting the base remains conductive due to the fixed charges. Since very little current goes through the system, the whole base has the same potential, which give rise to the polarization visible in Figure 3d. By increasing $V_{E B}$ to $3 \mathrm{~V}$, cations are injected from the base into the junction where they are compensated by anions from the emitter. The formation of a linear concentration gradient (Figure 3e,f) is the result of trapping of cations by the emitter and collector. Anions are injected into the junction from the emitter and diffuse over the junction to the collector where they are extracted. Cations, however, are trapped and are not transported through the junction. In order to keep the gradient of cations in place, a small potential gradient is necessary within the junction to cancel out the diffusional flux of cations. Thus, the transport of anions is the result of equal contributions by diffusion and migration. The current through the system causes ohmic potential losses in the emitter and collector, thus the potential gradient is more evenly distributed in this active mode than in the cutoff mode (Figure $3 \mathrm{~g}$ ).

The steady state is achieved when the base current compensates for the leakage of cations out of the junction. This leakage is typically small, and thus, steady state occurs approximately when the potential difference between the base and junction vanishes. Therefore the potential over the emitter approximately equals $V_{E B}$, which gives rise to the linear relationship in the transfer curve. The salt concentration within the junction is regulated by the base and thus depends on the current through the system. However, since the potential losses of the emitter and collector are equal and typically do not sum up to $V_{E C}$, the additional potential is lost somewhere in the device. This occurs in the extended space charge layer at the junction-collector interface which is described in more detail below (Figure 7).

The transition from the cutoff mode to the active mode occurs at $V_{E B}=V_{T}$ which is referred to as the threshold voltage. The threshold voltage for the simulated transfer curve in Figure 2 is $V_{T}=-0.25 \mathrm{~V}$, which is very close to the measured threshold voltage. This confirms that the measured threshold voltage is not an artifact of the experimental setup but rather a fundamental property of the system. As all currents are very small at the threshold voltage, its origin is likely due to the membrane potentials of the system. The membrane potential over a strong IEM depends on the concentration ratio of the two electrolytes surrounding the membrane ${ }^{8}$

$$
\Delta V_{\text {membrane }} \approx \frac{1}{z_{2} f} \ln \frac{\mathrm{c}^{\alpha}}{\mathrm{c}^{\beta}} .
$$

For $V_{E B}=V_{T}$ the ion concentration in the junction is approximately 200 times lower than the concentration of the emitter and base electrolytes. Because of the difference in selectivity of the emitter and base their membrane potentials have opposite signs. Thus, the total membrane potential between the emitter and base electrolytes is $\Delta V_{E J}-\Delta V_{J B} \approx(-0.13-0.13) \mathrm{V}=-0.26 \mathrm{~V}, \mathrm{a}$ value which is very close to the observed threshold 


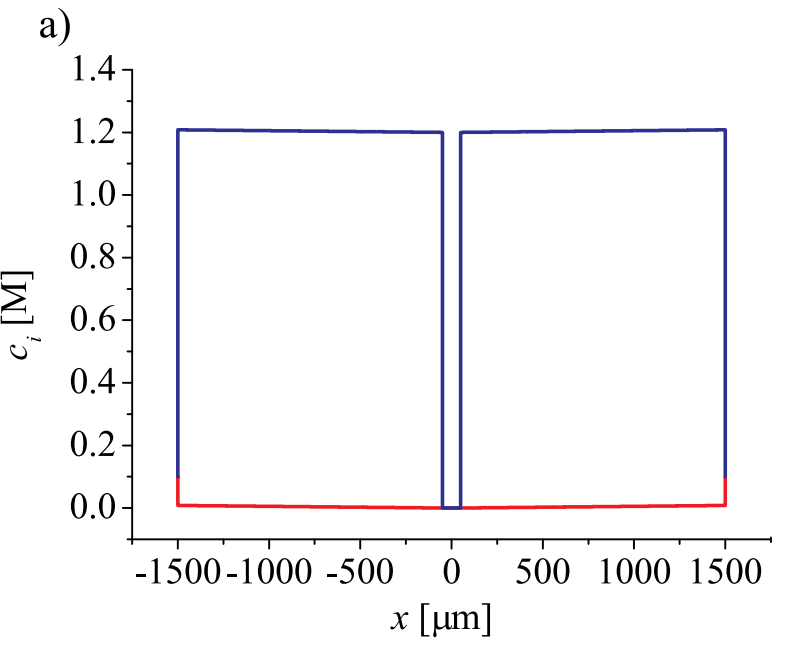

b)
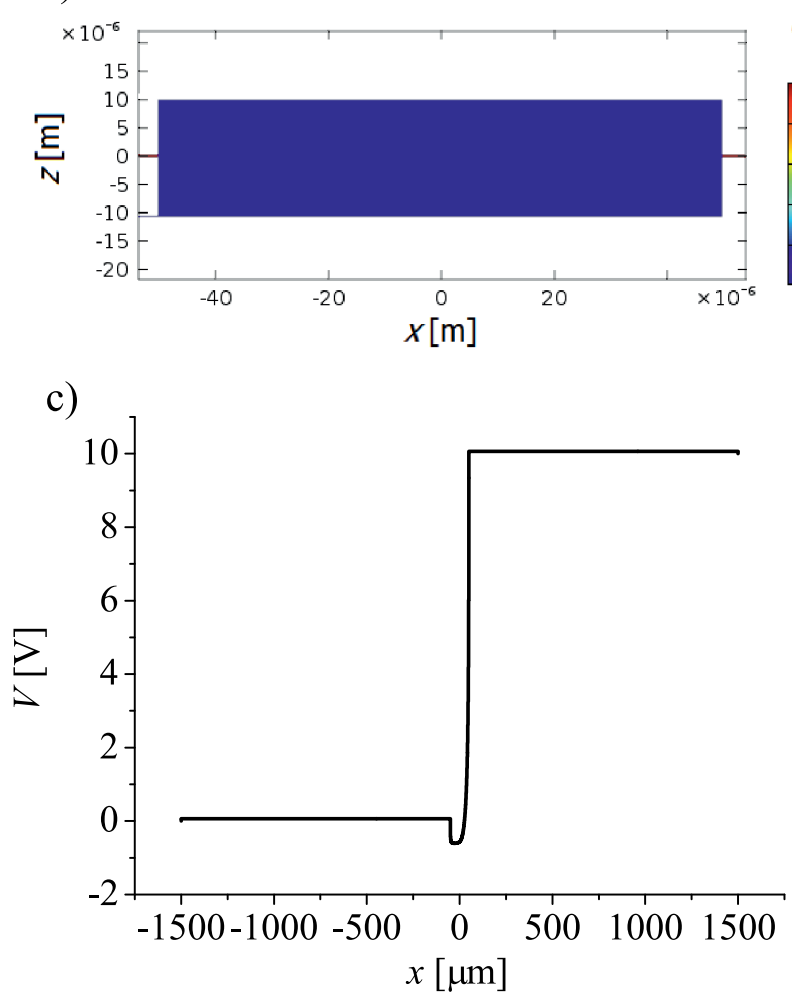

d)

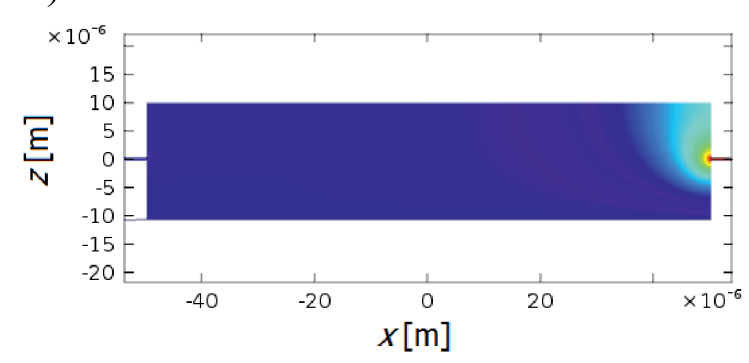

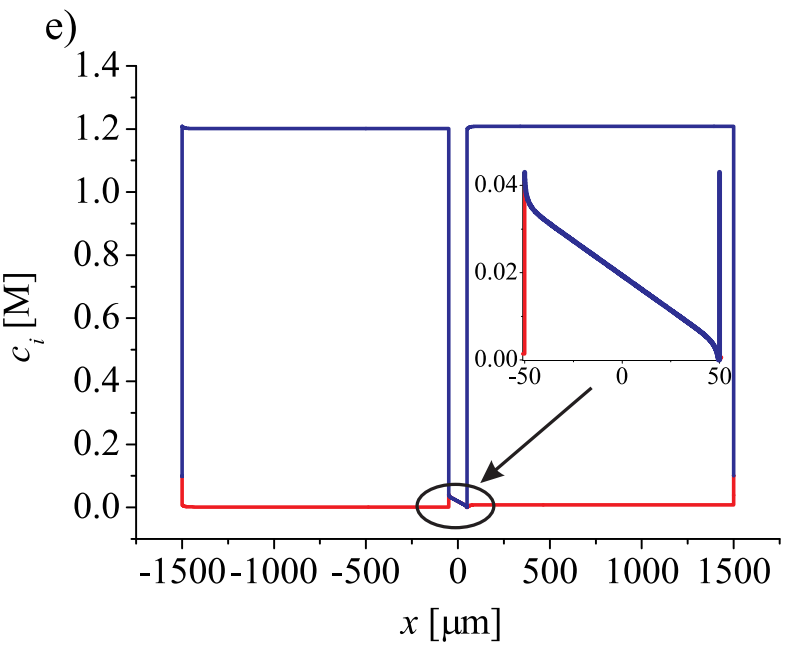

f)
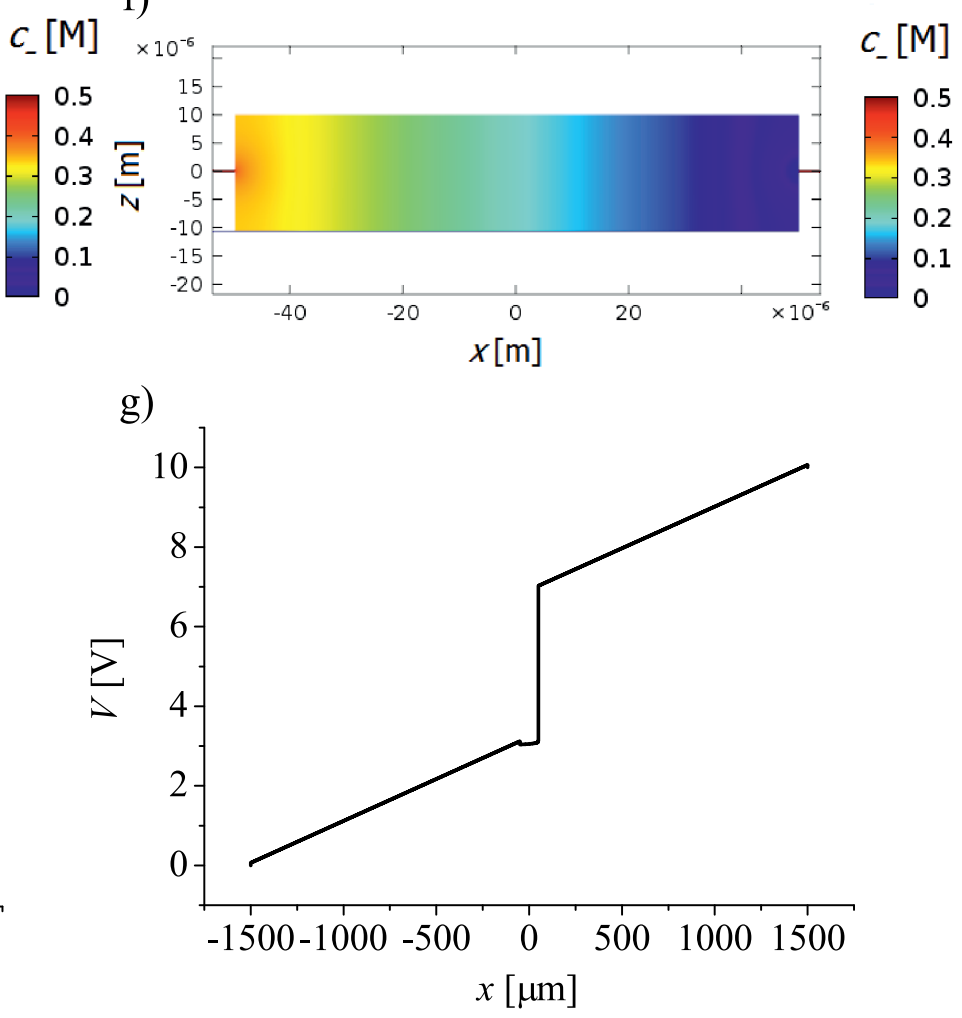

h)

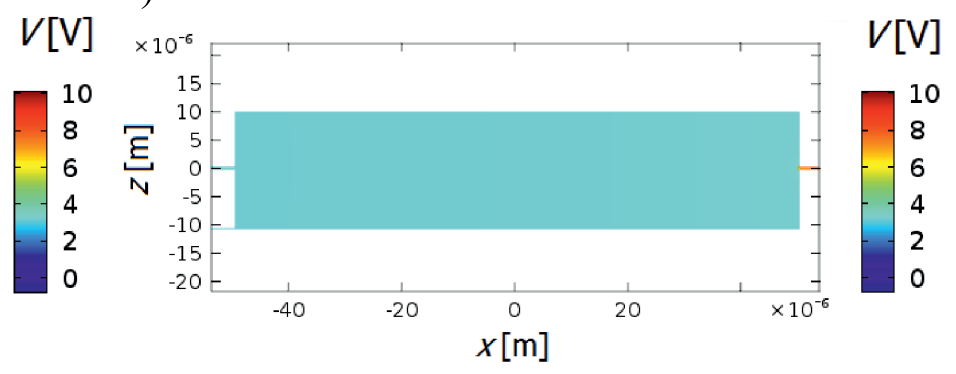

Figure 3: The concentration and potential profile in steady state for (a-d) cutoff mode $V_{E B}=-1 \mathrm{~V}$ and (eh) active mode $V_{E B}=3 \mathrm{~V}$, for $V_{E C}=10 \mathrm{~V}$. (a,e) Concentration profiles (blue line anions, red line cations) as a function of $x$ at $z=0$. (b,f) Anion concentration within the junction. (c,g) Potential profiles as a function of $x$ at $z=0$. (d,h) Potential maps of the junction. 


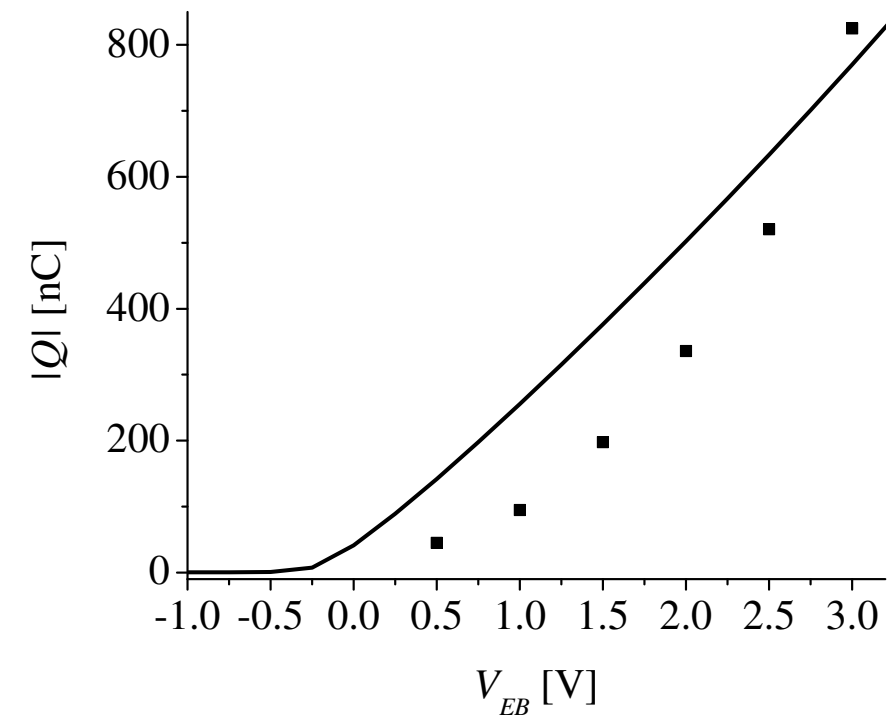

Figure 4: Total amount of anionic charge in the junction as function of the $V_{E B}$ for $V_{E C}=10 \mathrm{~V}$. The concentration of charge carriers is close to zero in cutoff mode but increases with higher $V_{E B}$.

voltage.

Figure 4 shows the simulated and measured amount of anionic charge within the junction for $-1<V_{E B}<3 \mathrm{~V}$ and $V_{E C}=10 \mathrm{~V}$. Only anions are considered as the amount of cations is virtually identical. Based on previous discussion one would expect a linear relationship between the amount of anions and the applied $V_{E B}$. The simulated curve is close to linear even though it bends off for lower $V_{E B}$. This behavior can be explained by the extension of the extended space charge layer, which is discussed later (Figure 7). The measured data, however, shows less linear behavior. This might be caused by a further extension of the extended space charge layer than predicted by the simulation. Another explanation might be that the effective diffusion coefficient decreases with an increased salt concentration.

Output characteristics. Figure 5 shows the simulated and measured output characteristics for $0<V_{E C}<10 \mathrm{~V}$ and $V_{E B}=-1 ; 1 ; 2 ; 3 \mathrm{~V}$. Three modes of operation are reveled in the graph. In saturation mode $I_{C}$ depends on $V_{E B}$ while in active and cutoff mode $I_{C}$ is independent of $V_{E B}$. The main features of the measured data are reproduced by our simulations; Although the measured $I_{C}$ evolution is not as flat in the active mode as achieved from the the simulated data. Also, the experimental data does not extend into the saturation regime because of device stability issues. In order to understand the shape of the output characteristics a few points, indicated by arrows in Figure 5, are studied in more detail in Figure 6.

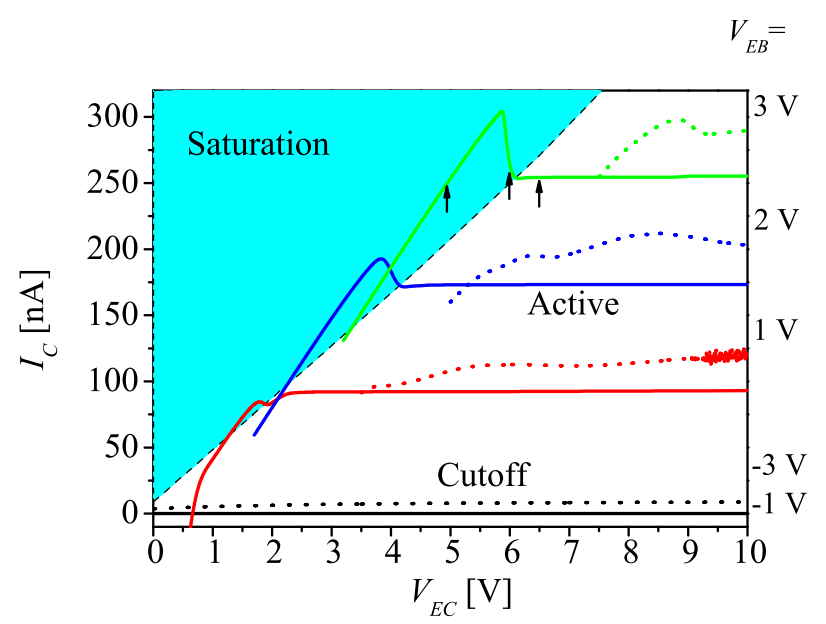

Figure 5: The output characteristics of the npnIBJT from numerical simulation (line) and experimental data (dot). ${ }^{10}$ The graph shows active, cutoff and saturation modes of the operation. The numerical dependence $I_{C}\left(V_{E C}\right)$ in cutoff mode was obtained for $V_{E B}=-1 \mathrm{~V}$ cause lower value of emitter-base voltage $\left(V_{E B}=-3 \mathrm{~V}\right)$ requires much more computational efforts. The concentration and potential profiles of the points indicated by arrows are given in Figure 6.

Far into the saturation regime $\left(V_{E B}=3 \mathrm{~V}\right.$ and $V_{E C}=5 \mathrm{~V}$ ) the base injects a large ionic current into the junction. This creates a very high salt concentration within the junction and the selectivity of the IEMs is lost as coions move into the channels. In an electronic BJT charge carriers can recombine and thereby reduce the carrier concentration. This is not possible with most ions and therefore steady state is achieved by diffusion of salt out from the junction. The concentrations predicted in Figure 6a are not physically reasonable within the materials, which might explain why failure of actual devices typically occurs if they are run in this mode. In real devices salt might also precipitate, something not accounted for in the presented model.

The transition from saturation to active mode is of great importance for the understanding of 

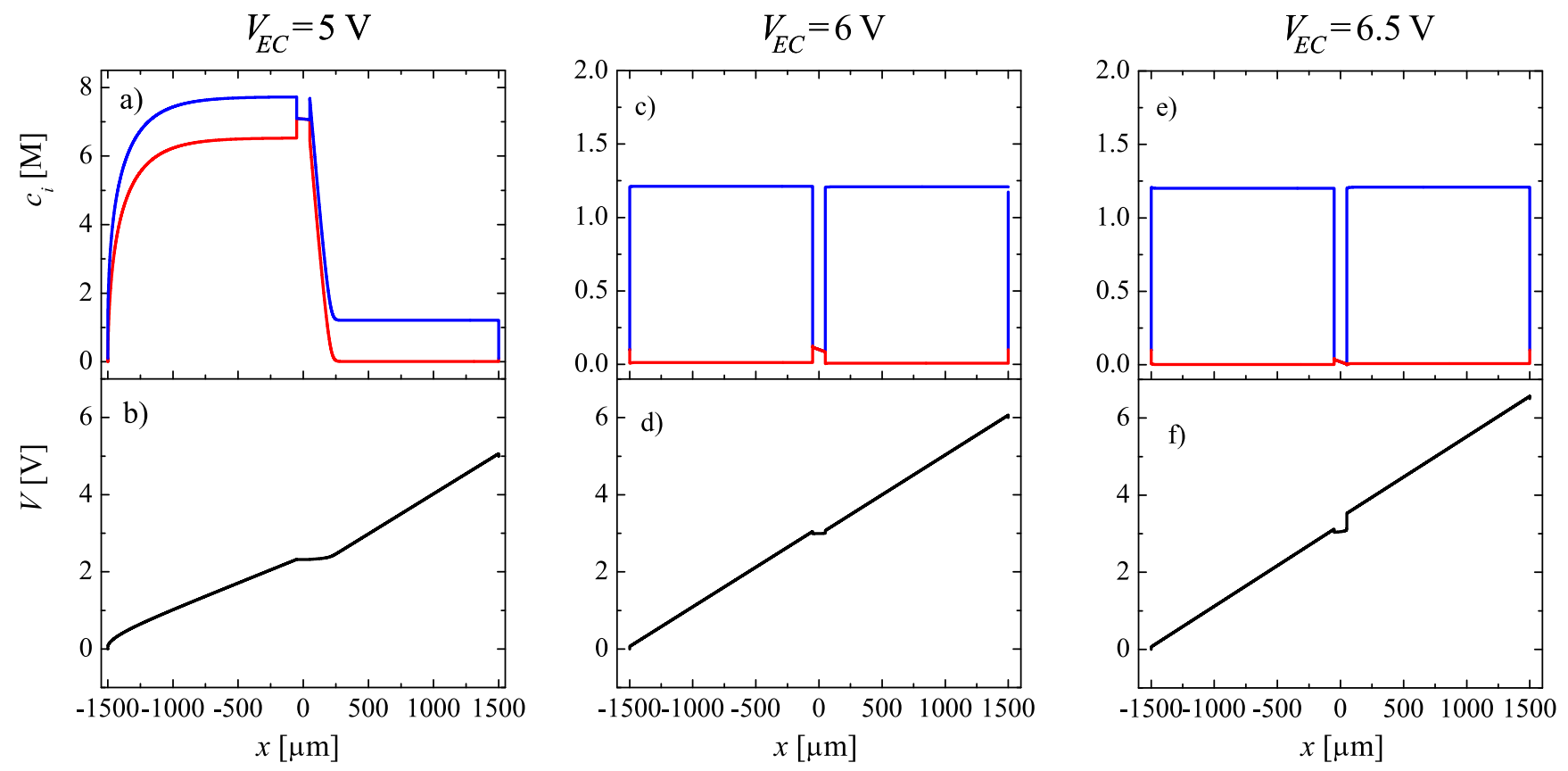

Figure 6: Concentration (blue line anions, red line cations) and potential profiles as function of $x$ at $z=0$ for a few chosen $V_{E C}$ voltages corresponded to arrows in Figure 5 and $V_{E B}=3 \mathrm{~V}$

device operation. When the operation goes towards active mode $\left(V_{E C} \approx 6 \mathrm{~V}\right)$ the base current decreases gradually. This reduces the leakage of coins (Figure 6a,c,e) and the salt concentration within the junction goes down. In saturation mode there is no significant potential drop at the junction-collector interface (Figure 6b,d). The transition from saturation (Figure 6c) to active mode (Figure 6e) occurs when the concentration at the junction-collector interface becomes close to zero. At this point the extended space charge layer starts to form with a distinct potential drop at the interface (Figure 6f). An overlimiting current is reached within the junction, in similarity to the overlimiting current of IEMs. ${ }^{16}$ When further increasing $V_{E C}$ and thereby moving deeper into active mode all the additional potential is lost at the junction-collector interface while $I_{C}$ remains constant. The cutoff mode is similar to the active mode as the operation is independent of $V_{E C}$ and the junction is depleted of all ions for every $V_{E C}$.

The extended space charge region (ESCR) at the junction-collector interface is of great importance to the operation of IBJTs as it accounts for a significant potential drop over a relatively short distance. The evolution of the ESCR for different applied voltages is shown in Figure 7. In Figure 7a the ESCR is plotted for varying $V_{E C}$ at constant $I_{C}$.
As the potential drop within the ESCR can be estimated to $V_{E C}-2 V_{E B}$, it is evident that the extension increases with the potential drop. In Figure $7 \mathrm{~b}$ the ESCR for different points along the transfer curve is shown. It should be noted that the extension is larger in this case because of the higher potential drop at the interface. The 2D shape of the ESCR is expected to be ellipsoidal some distance away from the collector, which is confirmed in Figure 7c. However, the width of the ESCR is quite small in all cases compared to the width between the emitter and collector. Thus one would expect that for these dimensions the variation in ESCR width should have little impact on the transistor characteristics. This would indicate that the measured nonlinear dependence in Figure 4 has other causes than variation in ESCR extension.

\section{Conclusions}

We have performed numerical modeling of the ion bipolar junction transistor which was developed and experimentally studied by Tybrandt et al. ${ }^{10}$ In order to describe the transport processes in the IBJT we used a 2D approximation based on the Poisson's-Nernst-Planck (PNP) equations. The model successfully reproduces the main ex- 
a)

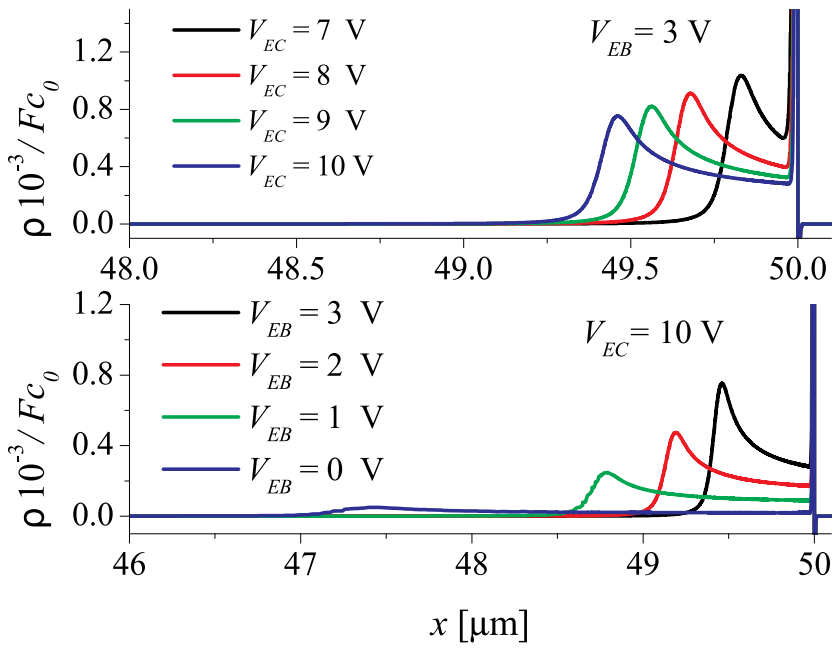

c)

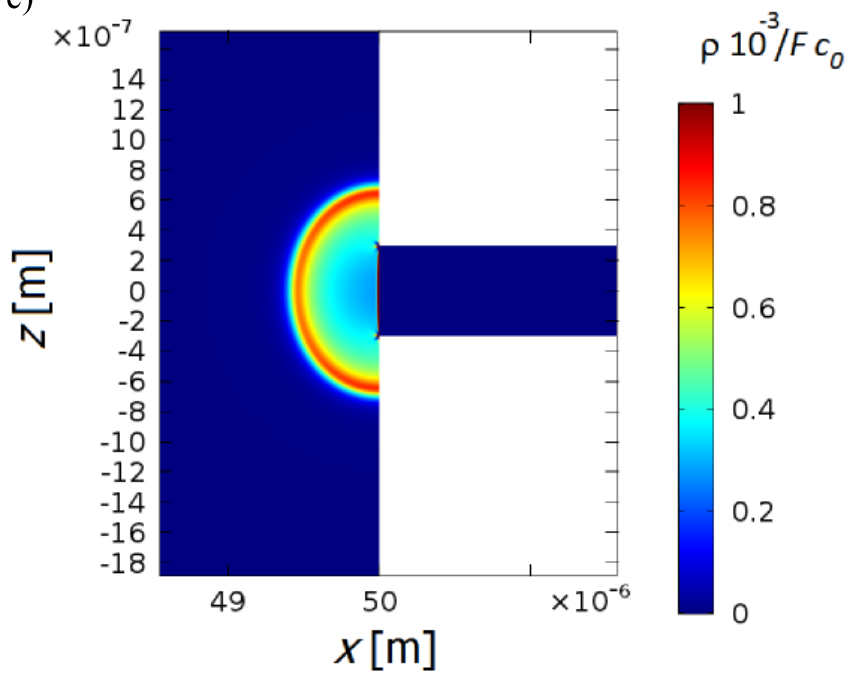

Figure 7: Space charge as a function of $x$ at $z=0$ for (a) different $V_{E C}$ and fixed $V_{E B}=3 \mathrm{~V}$; (b) for different $V_{E B}$ and fixed $V_{E C}=10 \mathrm{~V}$. (c) $2 \mathrm{D}$ plot of space charge region for $V_{E B}=3 \mathrm{~V}, V_{E C}=10 \mathrm{~V}$ in the active mode.

perimental device characteristics, i.e. the transfer curve and the output characteristics. Based on the detailed concentration and potential profiles provided by the model, the different modes of operation of the transistor were analyzed. The extremely high predicted concentrations in saturation mode can explain why real devices typically fail in this mode. The transitions between the different modes can be clearly understood from the concentration and potential profiles within the junction. The simulations also verifies that the experimentally found threshold voltage is not a measurement artifact and that it can be explained in terms of membrane potentials. The junction-collector interface was studied in detail because of its impor- tance to the understanding of the IBJT. It is concluded that a significant potential drop occurs at this interface which gives rise to an extended space charge region. The extension of this region varies with the potential drop, however the extension is always small compared to the distance between the emitter and collector. This can not cause the nonlinearity in Figure 4. Thus it is more likely that another effect, e.g. concentration dependent diffusion coefficients, is responsible for the nonlinearities found when measuring the amount of charge inside the junction. All in all the results provide the basis for a detailed understanding of IBJT operation and development. Specifically, the results indicate how critical device parameters like on-off ratio and switching speed can be improved. The dimensions of the device can be significantly reduced without being limited by the extension of the extended space charge region at the collector. This is good news as miniaturization of the IBJT is expected to improve switching speed. Further, a much better on-off ratio is predicted by the model than actually found in measurements. The difference is attributed to field enhanced water splitting, which is not accounted for in the model. Thus, by focusing on material modifications to reduce this kind of water splitting, further major improvements of this critical device parameter can be expected.

\section{Acknowledgement}

The research was supported by Norrköpings fond för Forskning och Utveckling, VINNOVA (OBOE - Strategic Research Center for Organic Bioelectronics), the Knut and Alice Wallenberg Foundation, the Royal Swedish Academy of Science, the Swedish Research Council (621-2008-2642) and the Önnesjö Foundation.

\section{References}

(1) Karnik, R.; Fan, R.; Yue, M.; Li, D.; Yang, P.; Majumdar, A. Electrostatic control of ions and molecules in nanofluidic transistors. Nano Lett. 2005, 5, 943-948 .

(2) Kim, K. B.; Han, J. H.; Kim, H. C.; Chung, T. D. Polyelectrolyte junction field effect 
transistor based on microfluidic chip. Appl. Phys. Lett. 2010, 96, 143506.

(3) Tybrandt, K.; Larsson, K. C.; RichterDahlfors, A.; Berggren, M. Ion bipolar junction transistors. Proc. Natl. Acad. Sci. U. S. A. 2010, 107, 9929-9932 .

(4) Priezjev, N. V.; Troian, S. Nanofluidics: Systems and applications. IEEE Sens. J. 2008, 8, 441-450 .

(5) Schoch, R. B.; Han, J. Y.; Renaud, P. Transport phenomena in nanofluidics. Rev. Mod. Phys. 2008, 80, 839-883 .

(6) Karnik, R.; Castelino, K.; Majumdar, A. Field-effect control of protein transport in a nanofluidic transistor circuit. Appl. Phys. Lett. 2006, 88, 123114 .

(7) Xu, T. W. Ion exchange membranes: State of their development and perspective. J. Membr. Sci. 2005, 263, 1-29 .

(8) Kontturi; K.; Murtomäki, L.; Manzanares, J. A. Ionic transport processes : in electrochemistry and membrane science. (Oxford University Press, 2008) .

(9) Mafe, S.; Ramirez, P. Electrochemical characterization of polymer ion-exchange bipolar membranes. Acta Polym. 1997 48, 234-250 .

(10) Tybrandt, K.; Gabrielsson, E. O.; Berggren, M. Toward Complementary Ionic Circuits: The npn Ion Bipolar Junction Transistor. $J$. Am. Chem. Soc. 2011, 133, 10141-10145 .

(11) Sze, S. Semiconductor devices: physics and technology. 2nd edn, (John Wiley \& Sons, 2002).

(12) Tybrandt, K.; Forchheimer, R.; Berggren, M. Logic gates based on ion transistors. Nat. Commun.2012, 3, 871 .

(13) Zabolotskii, V. I.; Manzanares, J. A.; Mafe, S.; Nikonenko, V. V.; Lebedev, K. A. Steadystate ion transport through a three-layered membrane system: A mathematical model allowing for violation of the electroneutrality condition. Russian Journal of Electrochemistry 2002, 38, 819-827 .

(14) Zabolotskii, V. I.; Lebedev, K. A.; Lovtsov, E. G. Mathematical model for the overlimiting state of an ion-exchange membrane system. Russian Journal of Electrochemistry 2006, 42, 836-846.

(15) Volgin, V. M.; Davydov, A. D. Ionic transport through ion-exchange and bipolar membranes. J. Membr. Sci. 2005, 259, 110-121 .

(16) Manzanares, J. A.; Murphy, W. D.; Mafe, S.; Reiss H. Numerical Simulation of the Non-equilibrium Diffuse Double Layer in Ion-Exchange Membranes. J. Phys. Chem., 1993, 97(32), 8524-8530 .

(17) Heywang, G.; Jonas, F. Poly(alkylenedioxythiophene)s - new, very stable conducting polymers. $A d v$. Mater. 1992, 4(2), 116-118 .

(18) Rubinstein, I.; Warshawsky, A.; Schechtman, L.; Kedem, O. Elimination of acid-base generation ('water-splitting') in electrodialysis. Desalination 1984, 51(1), 55-60 